\title{
Advanced Infrared Nanospectroscopy Using Photothermal Induced Resonance Technique, AFMIR: New Approach Using Tapping Mode
}

\author{
J. Mathurin*, A. Deniset-Besseau and A. Dazzi \\ Laboratoire de Chimie Physique (LCP), CNRS UMR 8000, Univ. of Paris-Sud, Université Paris-Saclay, \\ 91405 Orsay, France
}

For 10 years, a new instrumentation, called AFM-IR, has been developed in our team to perform IR analyses at the nanoscale. This technique is based on photothermal effect and is the result of a coupling between atomic force microscopy and IR spectroscopy to perform local IR absorption measurements. This combination makes it possible to identify at the nanoscale organic molecules without using exogenous markers. Nevertheless, in its initial configuration, the instrumentation has reached limits. New experimental approaches are therefore developed to solve the problems and overcome the technical constraints imposed by the system itself. In particular, two changes were significant: first, a change of configuration was done that allowed us to go down in terms of resolution and to reach tens of nanometers. The second concerned the AFM imaging itself: AFM-IR was used mainly in the contact mode, thus preventing its application in soft or loosely adhered. Here, we will describe this new mode which enabled addressing the limitations of the contact mode AFM-IR.

DOI: 10.12693/APhysPolA.137.29

PACS/topics: tapping-AFMIR, nanoscale infrared spectroscopy, photothermal technique

\section{Introduction}

In the last decade, a new instrumentation, called AFM-IR, was born at the Chemical-Physics laboratory to chemically identify samples at the nanometer scale. This technique is the result of a coupling between atomic force microscopy (AFM) and infrared (IR) spectroscopy (Patent US11/803421, A. Dazzi). The aim is to detect with the tip of the AFM in contact with the surface, the sample expansion induced within the sample after the IR radiation absorption [1-4]. This photothermal expansion occurring in the sample is a rapid process that forces the cantilever to oscillate on its eigenmodes. It was proved that the amplitude of the cantilever oscillations is directly linked to the absorbance $[1,4]$. This direct link allows to easily reconstruct the local IR absorption spectra and to perform chemical identification at the nanoscale organic molecules without using any exogenous markers. The main advantages in the detection of this process instead of a classical optical solution are to get rid of the wave-number dependence in terms of resolution as well as the limits imposed by the diffraction. The application fields range from biomedical to polymer science and astrochemistry.

Nevertheless, in its initial configuration, the instrumentation has reached limits in terms of sensitivity: the size of the smallest object (chemically pure) detectable is around $50 \mathrm{~nm}[1-3,5,6]$. New experimental approaches are therefore developed to solve the problems and overcome the technical constraints imposed by the system itself (configuration of illumination-sensitivitystudy of soft samples).

In particular, two changes were significant. First, a change of configuration was proposed that allowed going down in terms of resolution and to reach tens of nanometer $[7,8]$. This breakthrough has offered new perspectives such as the study of protein fibers or nanoparticles. The second concerned the AFM imaging itself: at first, AFM-IR was used in the contact mode, thus preventing its application in soft or loosely adhered samples such as polymeric nanoparticles (NPs) of less than $200 \mathrm{~nm}$. In those cases, the imaging mode called tapping is more appropriate: the cantilever oscillates on its fundamental mode and taps the surface. A so-called intermittent contact is maintained with the surface during the scanning process, limiting lateral shear forces and preserving the surface. The major challenge is the synchronization of the IR measurement with the AFM imaging intermittent contact mode.

Here, we present the main idea behind the tapping AFM-IR mode, that enables addressing the limitations of the contact mode AFM, thus extending its application to unravel the chemical and morphological features of soft, spherical samples difficult to fix on AFM substrate [9]. A short theoretical description of tapping-AFM-IR mode will then be proposed to highlight the physical parameters dominating this new method.

\section{Tapping AFM-IR concept}

For AFM imaging, the two most common modes are contact mode and oscillating mode with intermittent

\footnotetext{
jeremie.mathurin@csnsm.in2p3.fr
}

corresponding author; e-mail: 


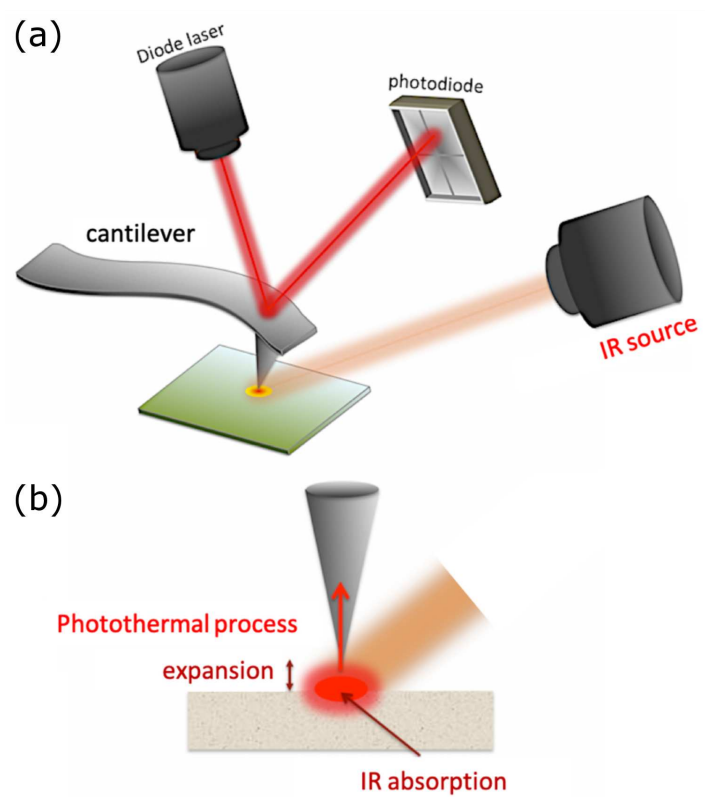

Fig. 1. (a) Schematic view of AFM-IR setup in contact mode. The IR illumination is from the top (b). Sketch illustrates the photothermal process.

contact called tapping mode. The AFM-IR technique was initially developed in contact mode as the IR measurement is done through the tip (see Fig. 1).

With the tapping mode, the lever is forced to oscillate constantly at a given amplitude and frequency. To implement an AFM-IR measurement with this AFM imaging mode, it is therefore essential to detect the oscillation generated by the photo-thermal expansion, whereas the cantilever is already oscillating. The solution takes place in a very well-known process: heterodyne detection.

This detection process already exists with AFM technique (referred as HFM [10]). The analysis of the amplitude and the phase of the generated heterodyne signal allow accessing the mechanical properties of the object.

Considering HFM techniques, a strategy for AFM-IR in tapping mode is thus proposed: the oscillation frequency of the tapping cantilever is mixed with the photothermal expansion frequency of the object. This is possible with the use of quantum cascade lasers (QCL) that possess a tunable repetition rate. As a consequence, the photothermal expansion of the object is adjustable to obtain a non-linear interaction as soon as the tip is in contact with its surface: in practice, the cantilever in tapping mode oscillates at the fundamental frequency (written mode 1, Fig. 2) and the frequency of the laser repetition rate is chosen so that the sum of these two frequencies corresponds to the frequency of the second cantilever mode (written mode 2, Fig. 2). The nonlinear interaction produced is therefore directly related to the photothermal expansion and will contain all the information related to the absorption of the object. The process is summarized in the schematic view given in Fig. 2.

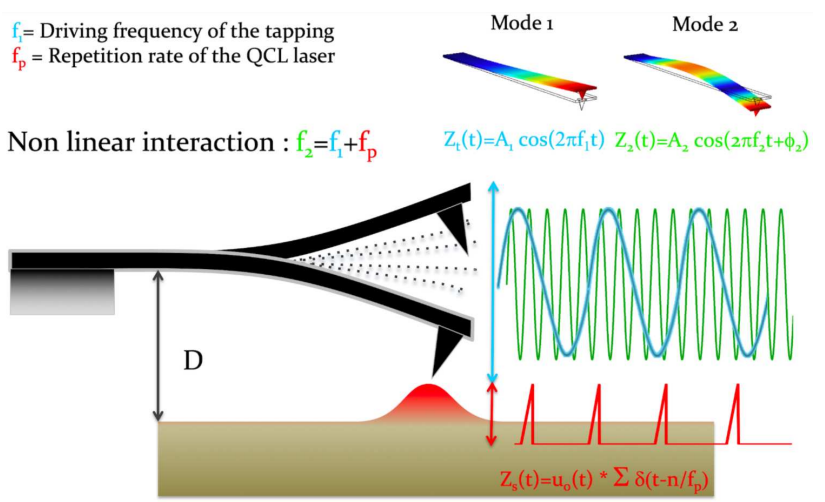

Fig. 2. Sketch illustrates the principle of tapping AFM-IR.

Experimentally, the protocol of the AFM-IR in tapping mode will be the following: before approaching the surface, the different modes of the cantilever are determined. The system performs a frequency sweep of the piezoelectric actuator over a frequency range between $10 \mathrm{kHz}$ and $1 \mathrm{MHz}$. Two modes are identified as frequencies $f_{1}$ (for the fundamental mode) and $f_{2}$ (for the 2 nd mode). The surface approach is done in a conventional manner with a feedback loop adjusted with the amplitude of the oscillations at $f_{1}$. Once the cantilever is in intermittent contact, the IR laser source is set to a repetition rate $f_{p}$ equal to $f_{2}-f_{1}$. To detect the IR absorption, the deflection signal is demodulated at the frequency $f_{2}$. As in contact mode, the topography and infrared measurements are intrinsically correlated.

\section{Theoretical background}

To describe the AFM-IR tapping mode, the fundamental mode $\left(\omega_{1}\right)$ is related to the topography of the surface (tapping) and the second mode $\left(\omega_{2}\right)$ to the infrared signal. The amplitude of the fundamental mode is $A_{1}$ and the cantilever-sample distance is $D$ (Fig. 3 ).

For each mode, the cantilever follows the following motion equations:

$$
\begin{aligned}
& \ddot{z^{\prime}}+\Gamma \dot{z^{\prime}}+\omega_{1}^{2} z^{\prime}=\frac{F_{\mathrm{ts}}(t)}{m_{1}^{*}}+\frac{F_{\text {piezo }}}{m_{1}^{*}} \cos \left(\omega_{1} t\right), \\
& \ddot{z}+\Gamma \dot{z}+\omega_{2}^{2} z=\frac{F_{\mathrm{ts}}(t)}{m_{2}^{*}},
\end{aligned}
$$

where $z^{\prime}$ is cantilever deflection for the first mode and $z$ is for the second mode, $\Gamma$ is damping factor, $m_{i}^{*}$ is mass of the $i$-th mode, $F_{\mathrm{ts}}$ is the interaction force between the tip and the surface and $F_{\text {piezo }}$ is excitation force used for the tapping.

The differential equation is solved in the Fourier domain. The expression of the deflection obtained from Eq. (2) gives directly the Fourier transform of the amplitude of the mode 2 , namely

$$
\tilde{z}(\omega)=\frac{\tilde{F_{\mathrm{ts}}}(\omega)}{m_{2}^{*}} \frac{1}{\omega_{2}^{2}-\omega^{2}+\mathrm{i} \Gamma \omega} .
$$




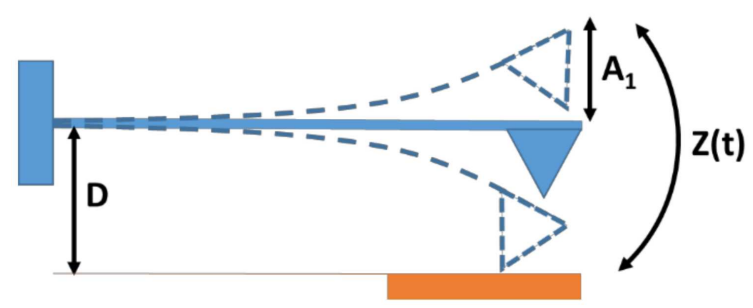

Fig. 3. Schematic view of the AFM using tapping mode.

Here $F_{\mathrm{ts}}$ might be described using two terms of the Hertzian contact

$$
F_{\mathrm{ts}}(t)=k_{z}\left(z^{\prime}(t)-D\right)^{3 / 2},
$$

where $k_{z}$ is the spring constant of the cantilever and $z^{\prime}(t)$ is the tip position.

During the tapping process $\left(z^{\prime}(t)\right), F_{\mathrm{ts}}$ is intermittent. We can define the following constant:

$$
\tau=\frac{2}{\omega_{1}} \arccos \left(\frac{D}{A_{1}}\right)
$$

that is the contact duration with the surface. The contact duration with the surface is directly linked to the force applied on the surface $D / A_{1}$.

To explain $F_{\mathrm{ts}}$, a rectangular function $\Pi(\tau)$ is introduced. For one interaction cycle, the cantilever oscillation $z_{\text {tap }}(t)$ is

$$
z_{\text {tap }}(t)=\Pi(\tau)\left[A_{1} \cos \left(\omega_{1} t+\varphi\right)-D\right],
$$

where $A_{1} \cos \left(\omega_{1} t+\varphi\right)$ is oscillation of mode 1 . In a consequence the interaction force becomes

$$
F_{\mathrm{ts}}(t)=k_{z}\left(z_{\text {tap }}(t)-u(t)\right)^{3 / 2} \text {. }
$$

Then a Taylor expansion is made

$F_{\mathrm{ts}}(t)=k_{z}\left(z_{\text {tap }}(t)-u(t)\right)+\chi_{s}\left(z_{\text {tap }}(t)-u(t)\right)^{2}+\ldots$,

where $\chi_{s}$ is the non-linear elasticity modulus of the surface and $u(t)$ is the photothermal expansion, i.e.:

$$
u(t)=B_{\mathrm{th}} \Delta T(t) \sum_{m=0}^{+\infty} \delta\left(t-m T_{0}\right) .
$$

Above $B_{\text {th }}=a G \alpha_{T}$ describes the thermal properties of the sample ( $a$ represent the size of the absorbing area, $G$ is a geometry-dependent constant, $\alpha_{T}$ is the coefficient of the thermal expansion of the sample), $\Delta T(t)$ is the temperature behavior after the IR absorption, and $T_{0}$ is repetition rate of the IR laser.

Looking at Eq. (8), the linear terms contribute to $\omega_{0}$ or $\omega_{1}$ and not to $\omega_{2}$. The square terms contribute to $2 \omega_{0}$ or $2 \omega_{1}$. Thus, only the double product gives a signal and $F_{\mathrm{ts}}$ :

$$
\begin{aligned}
& F_{\mathrm{ts}}(t)=-2 \chi_{s} z_{\mathrm{tap}}(t) u(t) \\
& F_{\mathrm{ts}}(t)=-2 \chi_{s} B_{\mathrm{th}} \Pi(\tau)\left[A_{1} \cos \left(\omega_{1} t+\varphi\right)-D\right] \\
& \quad \times \sum_{n=0}^{+\infty} \delta\left(t-n T_{1}\right) \Delta T(t) \sum_{m=0}^{+\infty} \delta\left(t-m T_{0}\right) .
\end{aligned}
$$

It is possible to simplify the expression of $F_{\mathrm{ts}}(t)$ if one considers that the interaction force is constant during the contact duration (true when the sample is rigid, $\left.k_{z} \gg k_{c}\right)$ :

$$
\begin{aligned}
& F_{\mathrm{ts}}(t)=-2 \chi_{s} \Pi(\tau)\left[A_{1}-D\right] \\
& \quad \times \sum_{n=0}^{+\infty} \delta\left(t-n T_{1}\right) B_{\mathrm{th}} \Delta T(t) \sum_{m=0}^{+\infty} \delta\left(t-m T_{0}\right) .
\end{aligned}
$$

Convolution of $F_{\mathrm{ts}}(t)$ with a Dirac comb of a $T_{1}$ period, $\sum_{n=0}^{+\infty} \delta\left(t-n T_{1}\right)$, allows to take the periodicity into account. Then in the Fourier domain

$$
\begin{aligned}
& \tilde{F_{\mathrm{ts}}}(\omega)=-2 \chi_{s}\left[2 \tau \frac{\sin \left(\omega \frac{\tau}{2}\right)}{\omega \tau}\left(A_{1}-D\right) \frac{2 \pi}{T_{1}} \sum_{n=0}^{+\infty} \delta\left(\omega-n \omega_{1}\right)\right] \\
& \times\left[u_{0} \frac{t_{p}}{2} \omega \sum_{0=0}^{+\infty} \delta\left(\omega-m \omega_{0}\right)\right] .
\end{aligned}
$$

Taking $\tilde{u}(\omega)=B_{\mathrm{th}} \tilde{T}(\omega)=B_{\mathrm{th}} T_{\max }\left(\frac{t_{p}}{2}+\tau_{\text {relax }}\right)$, one can see that $\tilde{u}(\omega)$ is a constant $u_{0}$.

Since the contact duration is very short compared to the period of the cantilever oscillations and the pulse duration, $\omega \tau$ approaches 0 :

$$
\begin{aligned}
& \tilde{F_{t s}}(\omega)=-\chi_{s} \tau\left(A_{1}-D\right) u_{0} t_{p} \omega_{1} \omega_{p}\left[\sum_{n=0}^{+\infty} \delta\left(\omega-n \omega_{1}\right)\right] \\
& \times\left[\sum_{m=0}^{+\infty} \delta\left(\omega-m \omega_{0}\right)\right] .
\end{aligned}
$$

Finally, $\tilde{F}_{\mathrm{ts}}(\omega)$ is the convolution product of two Dirac combs. To explain, let us consider the case $n=1$ and $m=1$. This case corresponds to the frequency sum (the difference is also possible but we do not use it during our experiments):

$$
\tilde{F}_{\mathrm{ts}}(\omega)=-\chi_{s} \tau\left(A_{1}-D\right) u_{0} t_{p} \omega_{1} \omega_{0} \delta\left(\omega-\left(\omega_{1}+\omega_{0}\right)\right) .
$$

Equation (13) is then inserted in Eq. (3):

$$
\begin{aligned}
& \tilde{z}(\omega)=\frac{-\chi_{s} \tau\left(A_{1}-D\right) u_{0} t_{p} \omega_{1} \omega_{0} \delta\left(\omega-\left(\omega_{1}+\omega_{0}\right)\right)}{m_{2}^{*}} \\
& \quad \times \frac{1}{\omega_{2}^{2}-\omega^{2}+\mathrm{i} \Gamma \omega} .
\end{aligned}
$$

Here $\tilde{z}(\omega)$ is maximum when $\omega_{1}+\omega_{0}=\omega_{2}$. The amplitude of the mode 2 is thus given by

$$
\|\tilde{z}(\omega)\|=\chi_{s} u_{0} \frac{\tau t_{p}\left(A_{1}-D\right)}{m_{2}^{*}} \frac{\omega_{1} \omega_{0}}{\Gamma \omega_{2}} .
$$

For $Q_{2}=\frac{\omega_{2}}{\Gamma}$ and $\omega=\omega_{2}-\omega_{1}$, one obtains

$$
\|\tilde{z}(\omega)\|=\chi_{s} u_{0} \frac{\tau t_{p}\left(A_{1}-D\right)}{m_{2}^{*}} \frac{\omega_{1}\left(\omega_{2}-\omega_{1}\right)}{\omega_{2}^{2}} Q_{2} .
$$

Furthermore, we know from previous work [4] the expression of

$$
\tilde{u}(\omega)=B_{\mathrm{th}} \tilde{T}(\omega)=B_{\mathrm{th}} T_{\max }\left(\frac{t_{p}}{2}+\tau_{\text {relax }}\right)
$$

and

$$
T_{\max }=\frac{t_{p}}{V \rho C_{p}} P_{\mathrm{abs}}=\frac{1}{4 \pi d} \ln (10) t_{p} \frac{I_{\mathrm{inc}} \alpha_{\mathrm{opt}} V}{V \rho C_{p}} A(\sigma)
$$


where $\rho$ is density of the sample, $C_{p}$ is its thermal capacity, $k_{\text {th }}$ is its thermal conductivity, $V$ is its volume, $\alpha_{\text {opt }}$ is its optical parameter, $\tau_{\text {relax }}$ is relaxation time, $d$ is the diameter of the absorbing object, and $I_{\text {inc }}$ is the incidence intensity (the resolution of this part could be found in the paper by Dazzi et al. [4]).

Finally, if $t_{p}<\tau_{\text {relax }}$ (true for the IR laser source used for our experiments):

$$
\begin{gathered}
\|\tilde{z}(\omega)\|=B_{\mathrm{th}}\left(\frac{t_{p}}{2}+\tau_{\text {relax }}\right) t_{p} \frac{I_{\mathrm{inc}} \alpha_{\mathrm{opt}} V}{V \rho C_{p}} \frac{\ln 10}{4 \pi d} \chi_{s} \\
\times \frac{\tau t_{p}\left(A_{1}-D\right)}{m_{2}^{*}} \frac{\omega_{1}\left(\omega_{2}-\omega_{1}\right)}{\omega_{2}^{2}} Q_{2} A(\sigma) .
\end{gathered}
$$

The final expression shows the link between $\|z(\omega)\|$ and the IR absorbance, $A(\sigma)$. Thus, the detected tapping AFM-IR signal is directly proportional to the IR absorption like the contact AFM-IR.

\section{Conclusion}

The new AFM-IR acquisition mode has been presented: the AFM-IR in tapping mode that enables AFMIR studies of soft or loosely adherent samples. The development of this advanced mode is based on a heterodyne detection. Indeed, a heterodyne signal is generated during the interaction of the cantilever oscillating at the fundamental mode frequency (mode 1) and the absorbing object, that expands during the illumination of the surface by the IR laser. To optimize the signal obtained, the laser repetition rate is tuned so that its sum with the first mode, used for the tapping, corresponds to the second mode of the cantilever.

The theoretical approach has illustrated the direct link between the observed signal and the IR absorbance of the object and identified the key parameters to optimize the IR signal. From these results, the AFM-IR interest in tapping mode was proved through two applications published recently $[11,12]$ : structural analysis of polymeric nanoparticles loaded with an antibiotic and investigation of organic matter heterogeneity in UCAMMs micrometeorites. Those studies have highlighted the tremendous breakthrough offered by this tapping AFM-IR technique but have also allowed us to identify some limitations, like the problems of measurement artifacts related to phase changes. Nevertheless, the quality of the results obtained may open a plethora of applications especially in material science.

\section{References}

[1] B. Lahiri, G. Holland, A. Centrone, Small 9, 439 (2013).

[2] A. Dazzi, C.B. Prater, Q. Hu, D.B. Chase, J.F. Rabolt, C. Marcott, Appl. Spectrosc. 66, 1365 (2012).

[3] A. Dazzi, C.B. Prater, Chem. Rev. 117, 5146 (2017).

[4] A. Dazzi, F. Glotin, R. Carminati, J. Appl. Phys. 107, 1 (2010).

[5] A. Dazzi, C.B. Prater, in: Infrared Spectroscopy, Book 2, Chapter Number AFM-IR, 2011, p. 1,.

[6] C. Mayet, A. Dazzi, R. Prazeres, F. Allot, F. Glotin, J.M. Ortega, Opt. Lett. 33, 1611 (2008).

[7] F. Lu, M.A. Belkin, Opt. Expr. 19, 19942 (2011).

[8] J.R. Felts, H. Cho, M.F. Yu, L.A. Bergman, A.F. Vakakis, W.P. King, Rev. Sci. Instrum. 84, 023709 (2013).

[9] J. Mathurin, E. Pancani, A. Deniset-Besseau, K. Kjoller, C.B. Prater, R. Gref, A. Dazzi, Analyst 143, 5940 (2018).

[10] M. Cuberes, H.E. Assender, G.A.D. Briggs, O.V. Kolosov, J. Phys. D Appl. Phys. 33, 2347 (2000).

[11] D. Partouche, J. Mathurin, A. Malabirade, S. Marco, C. Sandt, V. Arluison, A. Deniset-Besseau, S. Trépout, J. Microsc. 274, 23 (2019).

[12] J. Mathurin, E. Pancani, A. Deniset, K. Kjoller, C. Prater, R. Gref, A. Dazzi, Analyst 143, 5940 (2018). 